\title{
Epidemiological and Microbiological Analysis of Ventilator-Associated Pneumonia Patients in a Public Teaching Hospital
}

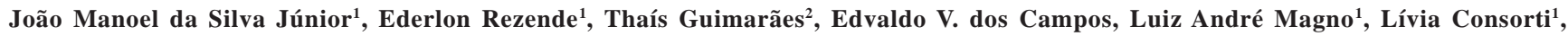
Renata Andréa Pietro Pereira ${ }^{1}$, Maria de Lourdes Nascimento ${ }^{2}$ and João Silva de Mendonça ${ }^{2}$

${ }^{1}$ Intensive Care Department; ${ }^{2}$ Infectious Diseases Department, Hospital do Servidor Público Estadual de São Paulo (HSPE-SP); São Paulo,
\end{abstract} SP, Brazil

\begin{abstract}
Ventilator-associated pneumonia (VAP) is the most commonly-acquired infection in patients in intensive care units. We analyzed epidemiological and microbiological characteristics and the outcome, in a cohort of criticallyill patients with confirmed diagnosis of VAP. All patients who had been on mechanical ventilation (MV) for more than 48 hours were included in our study; material collection for microbiological analysis was done within the first 24 hours after beginning treatment or after changing antibiotics. There were 55/265 (20.7\%) VAP cases diagnosed, at a rate of 21.6 episodes per 1,000 days of mechanical ventilation. Mean age of the patients was 66 years, with a mean APACHE II score of $26.7+7.0$; male patients were more prevalent. The mortality rates in the intensive care unit (ICU) and during the hospital stay were $71 \%$ and $80 \%$, respectively. MV duration in patients with VAP was 17 (range 3-43) days and among patients who had not developed VAP, $6(2-32)$ days $(\mathbf{p}<0.0001) .98 .2 \%$ of the samples were positive, with a high prevalence of Gram-negative bacteria, mainly Acinetobacter calcoaceticus. Risk factors for death included age, MV duration and surgery. VAP incidence in this sample of critically-ill patients was high, with a high mortality rate. Control and prevention strategies based on continuing education of healthcare workers, developed by a multidisciplinary team, should be encouraged to minimize morbimortality of this infection.
\end{abstract}

Key-Words: Ventilator-associated pneumonia, epidemiology, microbiology.

Ventilator-associated pneumonia (VAP) is the main cause of nosocomial infection in an intensive-care setting. VAP diagnosis is difficult, due both to the lack of well-defined clinical criteria and the overlap of symptoms with those of nosocomial pneumonia. American medical literature states that the mean incidence rate of VAP is 7 cases per 1,000 days of mechanical ventilation, ranging from 1 to over 20 cases per 1,000 days of mechanical ventilation [1,2]. VAP is the mainacquired infection during stay in an intensive care unit (ICU), diagnosed in up to $60 \%$ of patients. Its incidence varies from $23 \%$ to $28 \%$ in patients submitted to orotracheal intubation and mechanical ventilation, when we examine patients who do not have acute respiratory distress syndrome (ARDS) and $37 \%$ to $60 \%$ in patients with ARDS [3,4]. Mortality rates associated with this condition are high, from $25 \%$ to $50 \%$, and may reach $70 \%$ if the pulmonary infection is caused by multiresistant microorganisms [5,6].

Some predisposing factors, such as age, level of consciousness, trauma, burns, and the severity of the underlying disease are associated with a high risk for developing VAP; previous use of antibiotics and mechanical ventilation duration are the main factors involved. The highest rate of development of VAP occurs on about the fifth day of mechanical ventilation, reaching an incidence plateau after 15 days of mechanical ventilation, when the Received on 20 January 2007; revised 1 September 2007. Address for correspondence: Dr. João Manoel da Silva Júnior, M.D., Rua Pedro de Toledo, 1800 - 6 Andar - Vila Clementino - São Paulo - SP. Zip code: 04039-901, Brazil. Phone/Fax: (55-11) 5088-8146. Email: joaomanoel@sti-hspe.com.br.

The Brazilian Journal of Infectious Diseases 2007;11(5):482-488. (C) 2007 by The Brazilian Journal of Infectious Diseases and Contexto Publishing. All rights reserved. risk begins to fall, which explains the low rates of VAP observed in chronically-mechanically-ventilated patients [7].

Though an etiological investigation, composed of quantitative cultures from VAP patients (bronchoalveolar lavage fluid obtained through bronchoscopy with or without a protected catheter or simply collected by tracheal aspiration) is important for the optimization of antibiotic use [8], this recommendation is not universally accepted due to the lack of objective studies that demonstrate the benefit of these procedures [9]. There have been few trials made with criticallyill-patient populations concerning the importance of such data for clinical practice [10].

We examined the epidemiological and microbiological characteristics and the outcome of critically-ill patients submitted to mechanical ventilation for 48 hours or more and who have clinical diagnosis of VAP.

\section{Materials and Methods}

Ours was an observational, prospective study of a consecutive cohort of patients in a 20-bed ICU of a tertiary teaching hospital, conducted during eight months (from October 1, 2003 to May 31, 2004), after approval from the local ethics in research committee.

All patients in our hospital submitted to mechanical ventilation for more than 48 hours were included in the study. Clinical diagnosis of VAP was defined as the presence of a new and/or progressive lung infiltrate in a chest X-ray, associated with at least two of the following criteria: (1) purulent tracheal secretion; (2) white blood cell count $>12,000$ or $<$ $4,000 / \mathrm{mm}^{3}$ or bands count $>10 \%$; (3) axillary temperature $>$ $38^{\circ} \mathrm{C}$ or $<36^{\circ} \mathrm{C}$; and (4) worsening of $\mathrm{PaO}_{2} / \mathrm{FiO}_{2}$ ratio $>15 \%$ in the previous 48 hours. 
Patients with a low-survival expectancy in 30 days, who were diagnosed with acquired-immunodeficiency syndrome, were under chemotherapy, were agranulocytopenic or who were submitted to noninvasive-mechanical ventilation were excluded from the study.

All patients included in the study were submitted to the collection of demographic data and other variables useful to calculate Acute Physiology and Chronic Health Evaluation II (APACHE II) [11] and the Sequential Organ Failure Assessment (SOFA) [12] scores, considering the worst scores in the previous 24 hours before inclusion in the study. Sepsisconsensus conference criteria [13] were used to define organic dysfunction, and the worst results obtained 24 hours before inclusion in the study, as well as results from the microbiological examination were used to calculate the Clinical Pulmonary Infection Score (CPIS) [14]. All patients were followed up until discharge from the hospital.

All patients were submitted to material collection for culture (bronchoalveolar lavage fluid, tracheal aspirate and blood culture), within the first 24 hours after the beginning of antibiotic treatment or after changing antibiotics. Preferentially, fluid material was collected through bronchoalveolar lavage by bronchoscopy, due to the high sensibility and specificity of diagnosis of this type of material [15]. However, when it was not possible to submit patients to bronchoscopy within the first 24 hours after beginning a new antibiotic, material collection was done through tracheal secretion aspiration. Parameters used to define infection were: bronchoalveolar lavage fluid by bronchoscopy $>10^{4}$ colony-forming units (CFU)/mL and tracheal secretion aspiration $>10^{6} \mathrm{CFU} / \mathrm{mL}$ [16].

All patients included in our study had been empirically treated with antibiotics according to a standard protocol (Figure 1), elaborated by the ICU staff, together with the Infection Control Group, in February 2003; antibiotic therapy was considered adequate when the isolated microorganism was found susceptible to the prescribed antibiotic based on examination of the bronchoalveolar lavage fluid by bronchoscopy or through tracheal-secretion aspiration.

The following routine-care procedures were maintained for all patients submitted to mechanical ventilation: nurse and physiotherapist assistance, use of a filter Heat-Moisture Exchanger (HME), tracheal aspiration in a closed system, oral hygiene with chlorexidine, supraglotic aspiration and semirecumbent position. Systematic change of respiratory circuits was not routinely performed.

Patients included in the study were compared to patients without diagnosis of lung infection in relation to mechanical ventilation duration; the similarity among groups was analyzed through APACHE II and SOFA scores. Data were expressed as mean + standard deviation, median, and interquartil interval or percentages. Quantitative variables with a normal distribution were analyzed with parametric methods (Student-T test) and qualitative variables or irregularlydistributed variables were analyzed with non-parametric methods (Chi-square, Fisher exact and Mann Whitney tests).
Statistical significance was considered when $\mathrm{p}<0.05$ (2-tailed).

The patients were classified as clinical or surgical patients. Antibiotic prophylaxis had been prescribed to all surgical patients. Surgeries were classified as elective or non-elective.

\section{Results}

During the study period, 496 patients were submitted to mechanical ventilation; 265 of these patients were submitted to mechanical ventilation for at least 48 hours, for a total of 2,546 days of mechanical ventilation. In the subgroup of patients submitted to mechanical ventilation for more than 48 hours, there were 55 cases of VAP (20.7\%), with a rate of 21.6 cases per 1,000 mechanical ventilation days.

The duration of mechanical ventilation in patients with VAP was 3 to 43 days, with median of 17 days. In patients without diagnosis of VAP, mechanical ventilation duration was 2 to 32 days, with median of 6 days ( $<<0.0001$, Figure 2 ). The main demographic characteristics of the patients who developed VAP are presented in Table 2. Mean age was 66 years, with a prevalence of male patients and ICU and hospitalmortality rates were $71 \%$ and $80 \%$, respectively.

At diagnosis of VAP, patients had been in the hospital, in the ICU and/or had been on mechanical ventilation for long periods. Moreover, VAP patients had longer total hospital and ICU stays, as well as lengthier total mechanical ventilation periods (Table 3).

Among 55 patients with a clinical diagnosis of VAP, all but one was found to be infected based on microbiological analysis of bronchoalveolar lavage fluid or tracheal secretion, with a high prevalence of Gram-negative rods, mainly Acinetobacter calcoaceticus. Among blood cultures, only $58 \%$ showed growth of any microorganism (Table 4).

Univariate analysis of patients with VAP demonstrated the following risk factors for mortality: age, mechanical ventilation duration and surgery. Clinical patients had significantly lower mortality rates. There was higher mortality among patients with inadequate empirical antibiotic therapy; however, this difference was not significant (Table 5).

Outcome analysis of patients with positive blood cultures gave no significant differences, with 28/44 (64\%) deaths and 4/11 (36.4\%) hospital discharges ( $p=0.09)$.

Clinical patients had both shorter ICU and hospital length of stay before VAP diagnosis, and lower prevalence of inadequate empirical antibiotic therapy than did surgical patients; this was especially evident among patients transferred from the emergency room. This may be the reason for the lower mortality rate in this group of patients (Table 6).

\section{Discussion}

VAP is the most common nosocomial infection in patients who need mechanical ventilation [1]; the cumulative risk of developing pneumonia is $1 \%$ daily in these patients [9]. We found a $21 \%$ VAP incidence of, with longer mechanical ventilation duration in patients who developed infection, compared to patients without infection. 
Figure 1. Standard protocol for empirical therapy of patients on mechanical ventilation (MV) who develop ventilator-associated pneumonia (VAP) at the Hospital do Servidor Público Estadual de São Paulo at the time of teh study.

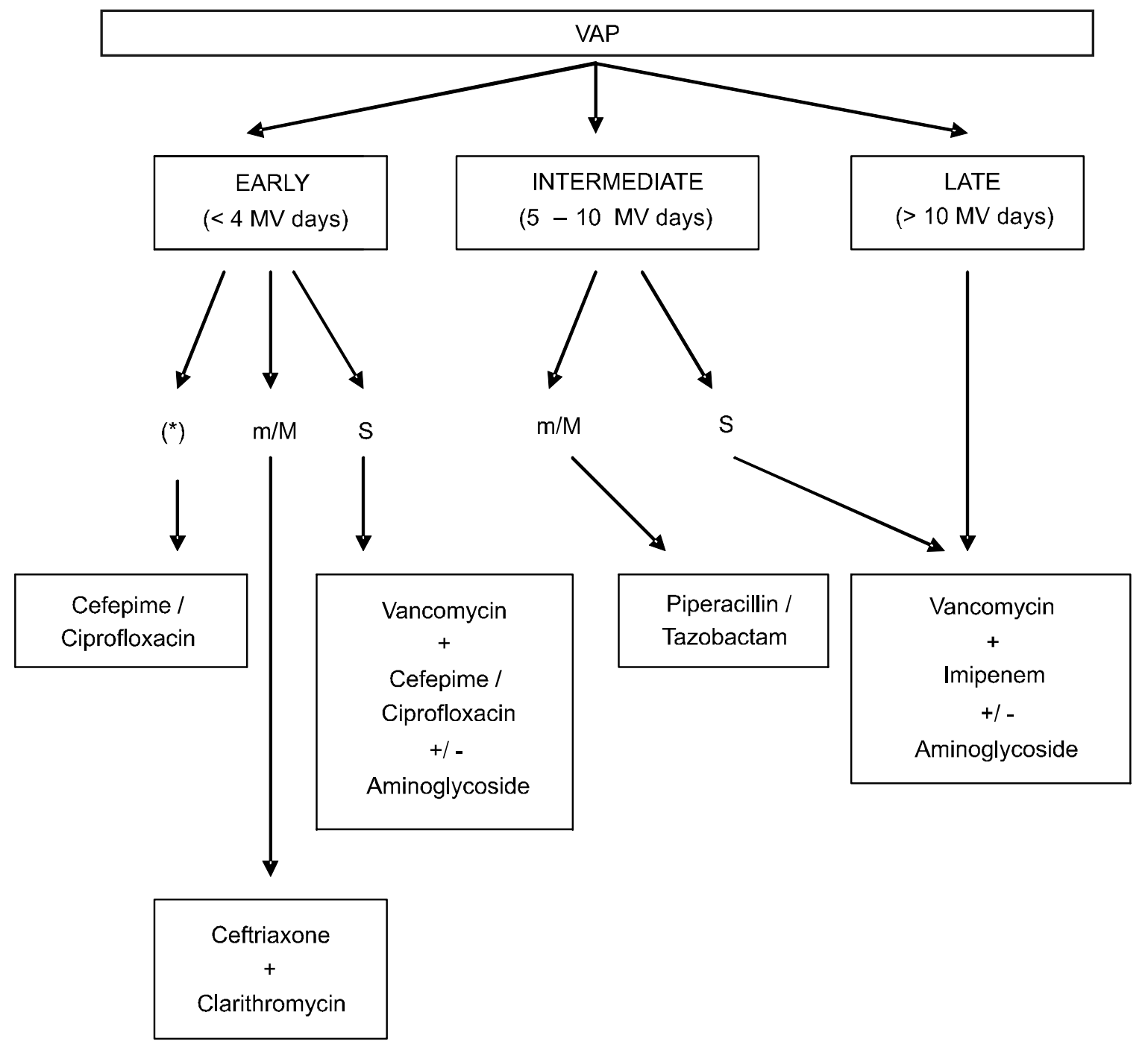

$\left({ }^{*}\right)>4$ days in hospital; $\mathrm{m} / \mathrm{M}=$ mild / Moderate; $\mathrm{S}=$ severe

Table 2. Demographic data of ventilator-associated pneumonia patients in the intensive care unit of the Hospital do Servidor Público Estadual de São Paulo

\begin{tabular}{lc}
\hline Variables & Characteristics \\
\hline Age (years) & $66.1+12.7$ \\
Male (\%) & 69.1 \\
Surgical patients (\%) & 50.9 \\
Clinical patients (\%) & 49.1 \\
APACHE II score & $26.7+7.0$ \\
SOFA score & $9.2+3.3$ \\
CPIS score & $9.0+1.8$ \\
\hline
\end{tabular}

Table 3. Time, in days, that the patients spent in the intensive care unit (ICU), in the hospital and on mechanical ventilation

\begin{tabular}{lc}
\hline Periods & $\begin{array}{c}\text { Mean + Standard } \\
\text { deviation }\end{array}$ \\
\hline Hospitalized before VAP diagnosis & $18.7+16.1$ \\
In the ICU before VAP diagnosis & $8.2+5.1$ \\
On mechanical ventilation before & $7.4+5.0$ \\
VAP diagnosis & $18.7+8.5$ \\
Mechanical ventilation duration & $23.2+10.0$ \\
In the ICU (total) & $41.3+24.7$ \\
In the Hospital (total) & \\
\hline VAP=ventilator-associated pneumonia. &
\end{tabular}


Table 4. Prevalence of microorganisms isolated from bronchoalveolar lavage fluid, tracheal secretion and blood cultures in patients diagnosed with ventilator-associated pneumonia in the intensive care unit of the Hospital do Servidor Público Estadual de São Paulo

\begin{tabular}{lccc}
\hline & $\begin{array}{c}\text { Bronchoalveolar lavage } \\
\text { fluid (\%) (N/ total) }\end{array}$ & $\begin{array}{c}\text { Tracheal aspirate (\%) } \\
\text { (N/ total) }\end{array}$ & $\begin{array}{c}\text { Blood cultures (\%) } \\
\text { (N/ total) }\end{array}$ \\
\hline Total prevalence & $49 / 50(98)$ & $46 / 50(92)$ & $32 / 55(58)$ \\
Acinetobacter calcoaceticus & $26 / 49(53)$ & $25 / 46(54)$ & $9 / 32(28)$ \\
Pseudomonas aeruginosa & $13 / 49(27)$ & $11 / 46(24)$ & $5 / 32(16)$ \\
Staphylococcus aureus & $0 / 49(0)$ & $2 / 46(4.3)$ & $6 / 32(19)$ \\
Enterobacteria* & $9 / 49(18)$ & $6 / 46(13)$ & $9 / 32(28)$ \\
\hline
\end{tabular}

* Enterobacteria include: Klebsiella pneumoniae, Serratia marcences, Providencia stuart, Enterobacter sp. and Proteus sp.

Table 5. Univariate analysis of characteristics of ventilator-associated pneumonia (VAP) patients divided according to mortality status

\begin{tabular}{lccc}
\hline Variables & Died (N = 39) & Alive (N = 16) & p value \\
\hline Age (years) & $69.4+9.5$ & $58.1+16.1$ & 0.002 \\
APACHE II score & $26.8+6.9$ & $26.4+7.4$ & NS \\
SOFA score & $9.0+3.4$ & $9.5+3.3$ & NS \\
CPIS score & $8.9+1.8$ & $9.1+1.9$ & NS \\
Mechanical ventilation duration (days) & $20.6+8.5$ & $14.1+6.8$ & 0.009 \\
Mechanical ventilation duration before VAP diagnosis (days) & $7.4+4.1$ & $7.6+6.5$ & NS \\
ICU length of stay (days) & $23.7+10.1$ & $21.7+10.0$ & NS \\
Hospital length of stay (days) & $40.7+24.9$ & $42.6+24.9$ & NS \\
ICU length of stay before VAP diagnosis (days) & $8.2+4.6$ & $8.3+6.3$ & NS \\
Hospital length of stay before VAP diagnosis (days) & $19.5+15.4$ & $16.7+18.0$ & NS \\
Male (\%) & 64.1 & 81.3 & NS \\
Clinical patients (\%) & 38.5 & 75.0 & 0.030 \\
Surgical patients (\%) & 61.5 & 25.0 & 0.030 \\
Elective surgical patients (\%) & 35.9 & 25.0 & 0.641 \\
Non-elective surgical patients (\%) & 25.6 & 0.0 & 0.094 \\
Inadequate empirical antibiotic therapy (\%) & 35.9 & 18.8 & $\mathrm{NS}$ \\
Need of changing antibiotic (\%) & 79.5 & 62.5 & $\mathrm{NS}$ \\
\hline
\end{tabular}

NS=not significant; ICU=intensive care unit.

Table 6. Number of days that patients were in the hospital until ventilator-associated pneumonia diagnosis and number of patients with inadequate empirical antibiotic therapy

\begin{tabular}{lccc}
\hline Variables & $\begin{array}{r}\text { Clinical } \\
\text { patients } \\
(\mathbf{N}=\mathbf{2 7})\end{array}$ & $\begin{array}{c}\text { Surgical } \\
\text { patients } \\
\mathbf{( N = 2 8 )}\end{array}$ & Pvalue \\
\hline ICU length of stay* $^{*}$ & $6(5-12)$ & $7(4.2-12)$ & 0.05 \\
Hospital length of stay* & $11(6-21)$ & $18(7.5-26.2)$ & 0.006 \\
Inadequate empirical & 5 & 12 & 0.008 \\
antibiotic therapy & & & \\
\hline
\end{tabular}

* Median (25-75 percentile). ICU=intensive care unit.
Figure 2. Number of days on mechanical ventilation for patients with and without ventilator-associated pneumonia (VAP) diagnosis.

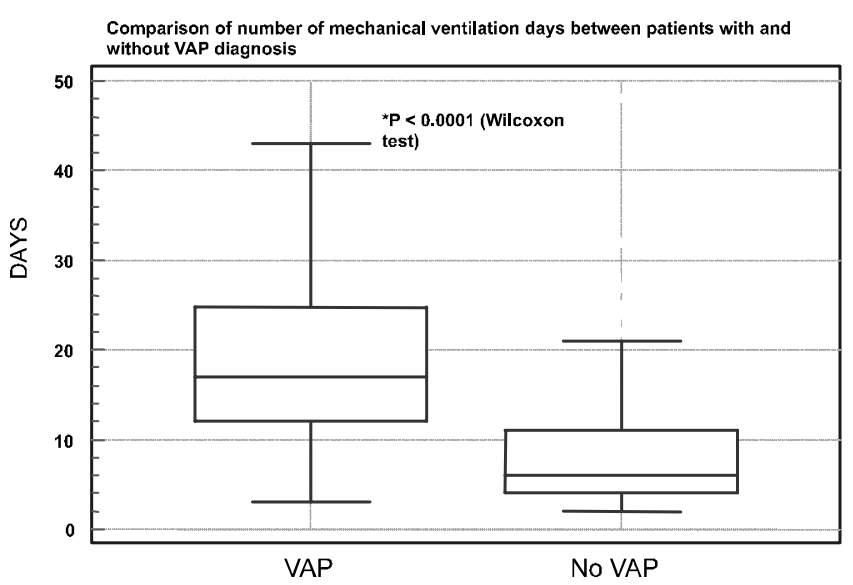


A European study [17] demonstrated that pneumonia is the leading cause of acquired infection in the ICU, with a prevalence of $46.9 \%$. In the United States, another study with VAP patients found a 9.3\% incidence rate [18].

VAP is the most prevalent nosocomial infection; its high incidence in our sample could be a consequence of various factors, such as the severity of the underlying disease of patients included in the study (APACHE II score above 16), advanced age and prolonged mechanical ventilation duration. These factors, associated with the need for food administration through a nasogastric tube and prolonged sedation [19] are associated with a higher risk of developing VAP. In Brazil, epidemiological data on the incidence and prevalence of VAP in an ICU setting are rare, but some studies have demonstrated high prevalence rates (about 20\% to 40\%) [20,21].

In our study, ICU and hospital mortality rates were 71 and $80 \%$, respectively. The risk of death in patients with ICUacquired pneumonia is 2 to 10 times higher [22,23]. Although some analyses indicate that VAP is a severe disease, it has not yet been clearly demonstrated that pneumonia is a factor responsible for increased mortality, due to the difficulty in making the diagnosis, divergent clinical criteria among studies and lack of a standard test that allows objective diagnosis of this disease. However, necropsy data on 200 patients led to the conclusion that nosocomial pneumonia is responsible for $60 \%$ of fatal infections in hospitals, being the leading cause of death due to hospital-acquired infections [24].

In spite of the high mortality rate among VAP patients, we observed that the risk factors associated with a poor prognosis were age, mechanical ventilation duration and patients submitted to surgical interventions, especially non-elective surgeries. Clinical patients had lower mortality rates, especially clinical patients transferred to the ICU from the emergency room.

Advanced age clearly affects the outcome of VAP patients, since older patients do not have significant functional reserves and usually have an unfavorable outcome when they are severely infectioned. Some studies have shown that advanced age is an independent and important mortality factor in patients with severe infections [25,26]. Moreover, the longer the mechanical ventilation period, the longer the length of stay in the hospital and concurrently, the greater both the colonization rate and the incidence of resistant bacteria. There are reports $[27,28]$ of mortality rates of approximately $70 \%$ when resistant bacteria were involved in VAP.

The poor outcome in patients submitted to surgical interventions, especially non-elective surgeries, may be attributable to the fact that these surgeries can become contaminated, since these patients normally do not have adequate pre-surgical preparation. We found longer hospital stays and a higher prevalence of inadequate antibiotic therapy in this group of patients. On the other hand, clinical patients, especially those who were transferred from the emergency room, had shorter hospital stays and a lower prevalence of inadequate empirical antibiotic therapy, leading to lower mortality rates in this subgroup of patients.
Inadequate empirical antibiotic therapy choice increased mortality; however, this increase was not significant in our study. Changes in empirical antibiotic therapy were more common in patients who did not survive their disease episode. Many authors [22,29-32] have reported that initial inadequate antibiotic therapy is associated with poor prognosis and increased mortality rates, which ranged from $26 \%$ to $91 \%$ [22,29-32]. In critically-ill patients, such as those included in our study, inadequate antibiotic therapy may also result in decreased treatment efficacy, as well as delays in the administration of antibiotics.

Early introduction of broad-spectrum antibiotics, in patients with clinical suspicion of nosocomial pneumonia, decreases their bacterial load, minimizing risks and the potential devastating consequences of delays in therapy [33]. Clinical information about risk factors for the development of VAP, including comorbidities and previous use of antibiotics, may help to determine the best initial treatment choice.

Among the markers of disease severity, both APACHE II and SOFA scores did not differentiate patients who later survived from those who did not survive; consequently, different from previous studies [19], the mortality rates of our VAP patients were not influenced by the previous health status of these patients. Consequently, pneumonia appears to be crucial for these patients, as it provoked high mortality rates.

We found that clinical diagnostic criteria were useful for investigating VAP patients; once means CPIS score was 9.0. + 1.8. Though some authors consider CPIS scores higher than 6.0 to give $100 \%$ specificity for VAP diagnosis [14], we found mean values of CPIS scores markedly higher than 6.0. Besides clinical diagnosis, 98\% of the patients included in our study had confirmation of VAP through bacteriological culture of bronchoalveolar lavage fluid, tracheal secretion and/or blood.

We suggest that clinical-radiological criteria may be used for the initial clinical diagnosis of VAP and local knowledge of the most prevalent microorganisms should be used to guide empirical antibiotic therapy. Wunderink et al. [34] reported that clinical findings were good predictors for VAP diagnosis, based on eight studies.

Gram-negative rods were the most frequent microorganisms in the cultures; Acinetobacter calcoaceticus was the most common species. This bacterium has been responsible for about $50 \%$ of the nosocomial infections acquired in our ICU; mechanical ventilation duration, time in the ICU and in the hospital were positively correlated with VAP diagnosis. Consequently, time in the hospital before VAP diagnosis is useful for defining adequate empirical antibiotic therapy choices, like mechanical ventilation duration and previous use of antibiotic therapy [35].

Unexpectedly, we found a low prevalence of Staphylococcus aureus in the respiratory secretions of the patients; although this microorganism was present in 19\% among patients who had positive blood cultures. Nonfermenting Gram-negative bacteria can mask Gram-positive bacterial growth [36]; consequently, Staphylococcus aureus 
can become an important causative agent of VAP, especially in patients who have risk factors for VAP and are on mechanical ventilation for extended periods.

One of the greatest challenges in treating VAP patients is to establish an early and precise diagnosis. In patients with inadequate antibiotic therapy, delays in changing the therapeutic regimen can interfere unfavorably in the outcome of these patients [37]. When changes in antibiotics are based on early etiological diagnosis, patient outcome can improve [32]. The outcome of VAP depends on rapid identification of the causative microorganism; consequently, a well-equipped microbiology laboratory is essential.

Considering the high prevalence rates and high morbidity and mortality rates associated with VAP, prevention strategies are highly recommended and necessary. SENIC (Study on the Efficacy of Nosocomial Infection Control) concluded that about a third of all nosocomial infections could be prevented with adequate control and educational programs for healthcare workers [38].

After our study, we changed the empirical therapy protocol for VAP patients in the ICU of our hospital, adjusting treatment according to the susceptibility profile of the most-commonlyisolated microorganisms, also taking into account mechanical ventilation duration, previous use of antibiotics and risk factors for acquisition of multi-resistant pathogens.

In conclusion, VAP incidence in this cohort of critically-ill patients was high, and there was a high mortality rate; it was evident that this is an important disease for this group of patients. Advanced age, prolonged mechanical ventilation duration and surgery were risk factors associated with high mortality rates.

Though clinical diagnosis is adequate, bacterial confirmation is recommended based on knowledge of local epidemiological and susceptibility profiles. As inadequate empirical antibiotic therapy is common, and frequent changes are made from the initial antibiotic therapy, prevention and control programs based on continuing medical education of health care workers should be encouraged in order to minimize the morbimortality rates of VAP in critically-ill patients.

\section{Acknowledgements}

We thank André Gulinelli, M.D., for helping prepare this manuscript.

\section{References}

1. Richards M.J., Edwards J.R., Culver D.H., et al. Nosocomial infections in medical intensive care units in the United States. National Nosocomial Infections Surveillance System. Crit Care Med 1999;27:887-92.

2. Fagon J.Y., Chastre J., Domart Y., et al. Nosocomial pneumonia in patients receiving continuous mechanical ventilation. Am Rev Respir Dis 1989;139:877-84.

3. Chastre J., Trouillet J.L., Vuagnat A., et al. Nosocomial pneumonia in patients with acute respiratory distress syndrome. Am J Respir Crit Care Med 1998;157:1165-72.

4. Markowicz P., Wolff M., Djedaini K., et al. Multicenter prospective study of ventilator-associated pneumonia during acute respiratory distress syndrome. Am J Respir Crit Care Med 2000;161:1942-8.

5. Rello J., Valles J. Mortality as an outcome in hospital-acquired pneumonia. Infect Control Hosp Epidemiol 1998;19:795-7.

6. Fagon J.Y., Chastre J., Vuagnat A., et al. Nosocomial pneumonia and mortality among patients in intensive care units. JAMA 1996;275:866-9.

7. Cook D.J., Walter S.D., Cook R.J., et al. Incidence of and risk factors for ventilator-associated pneumonia in critically ill patients. Ann Intern Med 1998;129:433-40.

8. Hubmayr R.D. Statement of the 4th International Consensus Conference in Critical Care on ICU-Acquired Pneumonia Chicago, Illinois, May 2002. Intensive Care Med 2002;28:1521-36.

9. Ravindra M. Mehta and Michael S. Niederman. Nosocomial pneumonia. Curr Opin Infect Dis 2002;15:387-94.

10. Chastre J., Fagon J.Y. Ventilator-associated Pneumonia. Am J Respir Crit Care Med 2002;165:867-903.

11. Knaus W.A., Zimmerman J.E., Wagner D.P., et al. APACHE acute physiology and chronic health evaluation: a physiologically based classification system. Crit Care Med 1981;(9):591-7.

12. Vincent J.L., Mendonça A., Cantraine F., et al. Use of the SOFA score to assess the incidence of organ dysfunction/failure in intensive care units : results of a multicenter, prospective study. Crit Care Med 1998;26(11)1793-1800.

13. Bone R.C., Balk R.A., Cerra F.B., et al. Definitions for sepsis and organ failure and guidelines for the use of innovative therapies in sepsis. The ACCP/SCCM Consensus Conference Committee. American College of Chest Physicians/Society of Critical Care Medicine. Chest 1992;101(6):1644-55.

14. Pugin J., Auckenthaler R., Mili N., et al. Diagnosis of ventilatorassociated pneumonia by bacteriologic analysis of bronchoscopic and nonbronchoscopic 'blind' bronchoalveolar lavage fluid. Am Rev Respir Dis 1991;143:1121-9.

15. Marquette C.H., Copin M.C., Wallet F., et al.: Diagnostic tests for pneumonia in ventilated patients: prospective evaluation of diagnostic accuracy using histology as a diagnostic gold standard. Am J Respir Crit Care Med 1995;151:1878-88.

16. Torres A., Carlet J. Ventilator-associated pneumonia: European Task Force on ventilator associated pneumonia. Eur Respir J 2001; $17: 1034-45$.

17. Vincent J.L., Bihari D.J., Suter P.M., et al. The Prevalence of Nosocomial Infection in Intensive Care Units in Europe: Results of the European Prevalence of Infection in Intensive Care (EPIC) Study. JAMA 1995;274(8):639-44.

18. Rello J., Ollendorf D.A., Oster G., et al. Epidemiology and Outcomes of Ventilator-Associated Pneumonia in a Large US Database. Chest 2002;122:2115-21.

19. Hunter J.D., Corry P.R. Ventilator-associated pneumonia. BJA 2002;2(5):148-50.

20. Carvalho M.V.C.F., Winkeler G.F.P., Costa F.A.M., et al. Concordance between tracheal aspirate and bronchoalveolar lavage in the diagnosis of ventilator associated pneumonia. $\mathrm{J}$ Bras Pneumol 2004;30(1):26-38.

21. Balthazar A.B., Von N.A., Capitani E.M., et al. Diagnostic investigation of ventilator-associated pneumonia using bronchoalveolar lavage: comparative study with a postmortem lung biopsy. Braz J Med Biol Res 2001;34(8):993-1001.

22. Torres A., Aznar R., Gatell J., et al. Incidence, risk, and prognosis factors of nosocomial pneumonia in mechanically ventilated patients. Am Rev Respir Dis 1990;142:523-8.

23. Craven D., Kunches L., Kilinsky V., et al. Risk factors for pneumonia and fatality in patients receiving continuous mechanical ventilation. Am Rev Respir Dis 1986;133:792-6.

24. Gross P.A., Neu H.C., Aswapokee P., et al. Deaths from nosocomial infections: experience in a university hospital and a community 
hospital. Am J Med 1980;68:219-23.

25. Angus D.C., Linde-Zwirble W.T., Lidicker J., et al. Epidemiology of severe sepsis in the United States: Analysis of incidence, outcome, and associated costs of care. Crit Care Med 2001;(29):1303-10.

26. Mirzanejad Y., Roman S., Talbot J., et al. Pneumococcal bacteremia in two tertiary care hospitals in Winnipeg, Canada. Pneumococcal Bacteremia Study Group. Chest 1996;109:173-8.

27. Bryan C.S., Reynolds K.L. Bacteremic nosocomial pneumonia. Analysis of 172 episodes from a single metropolitan area. Am Rev Respir Dis 1984;129:668-71.

28. Fagon J.Y., Chastre J., Hance A.J., et al. Nosocomial pneumonia in ventilated patients: a cohort study evaluating attributable mortality and hospital stay. Am J Med 1993;94:281-8.

29. Heyland D.K., Cook D.J., Griffith L., et al. The attributable morbidity and mortality of ventilator-associated pneumonia in the critically ill patient. Am J Respir Crit Care Med 1999; $159: 1249-56$

30. Kollef M.H., Ward S. The influence of mini-BAL cultures on patients outcomes: implications for the antibiotic management of ventilator-associated pneumonia. Chest 1998; 113:412-420

31. Alvarez-Lerma F, and the ICU Acquired Pneumonia Study Group: Modification of empiric antibiotic treatment in patients with pneumonia acquired in the intensive care unit. Intensive Care Med 1996;22:387-94.

32. Luna C.M., Vujacich P., Niederman M.S., et al. Impact of BAL data on the therapy and outcome of ventilator-associated pneumonia. Chest 1997;111:676-85.

33. Iregui M., Ward S., Sherman C., et al. Clinical importance of delays in the initiation of appropriate antibiotic treatment for ventilator-associated pneumonia. Chest 2002;122:262-8.

34. Wunderink R.G. Clinical Criteria in the Diagnosis of VentilatorAssociated Pneumonia. Chest 2000;117:191S-4S.

35. Montero J.G., Ortiz-Leyba C., Hinojosa E.F., et al. Acinetobacter baumannii ventilator-associated pneumonia: epidemiological and clinical findings. Intensive Care Med 2005;31:649-55.

36. Kollef M.H. Antibiotic management of ventilator-associated pneumonia due to antibiotic-resistant Gram-positive bacterial infection. Eur J Clin Microbiol Infect Dis 2005;24(12):794803.

37. Meduri G.U., Mauldin G.L., Wunderink R.G., et al. Causes of fever and pulmonary densities in patients with clinical manifestations of ventilator-associated pneumonia. Chest 1994;106:221-35.

38. Haley R.W., Culver D.H., White J.W., et al. The efficacy of infection surveillance and control programs in preventing nosocomial infections in US hospitals. Am J Epidemiol 1985;121:182-205. 